\title{
Investigation into the_parameters of driver circuits for power electronic and DC-DC converters
}

\author{
Evgeni Malev ${ }^{1}$ \\ 1 - Technical University of Varna, Department of Electronics and Microelectronics, 9010, 1 Studentska Street, Varna, Bulgaria \\ Corresponding author contact: evgeni.maleveabv.bg
}

\begin{abstract}
The paper focuses exclusively on the_analysis of the simulation software and the main parameters of the driver circuits for power electronic converters. A mathematical model of the driver circuit in the Matlab Simunink software environment has been created. This model enables the analysis of the process of frequency formation through the boostrap capacitor and examines the interaction between the protection of the scheme at $\frac{1}{3} V_{c c}, \frac{1}{6} V_{c c}$ and the other digital blocks in the circuit. Considered, additionally, are the basic physical processes behavior of a driver with a similar structure in Infineon designer.
\end{abstract}

Keywords. MATLAB, simulation software, integrated circuit, DC - DC converter, driver circuit

\section{Introduction}

Electronic integrated circuits are experiencing rapid development. In the first integrated circuits, manufactured in early 1957, between 20 and 50 elementary logic blocks were crammed into an integrated circuit the size of a driver for a half-bridge converter $(8 \times 20 \mathrm{~mm})$. More than 1,000,000 logic elements can be assembled in modern integrated circuits in a housing of similar dimensions (Stievano et al., 2003) All in all, integrated manufacturing technology has evolved from Transistor-transistor logic (TTL) circuits to 3D design and Ultra Large Scale Integration (ULSI)(Lee \& Chakrabarty, 2009). Before being produced on an integrated circuit, various physical tests and analyzes are performed to determine its behavior in different modes of operation. Such physical tests and analyzes may be used to improve the integrated circuit or design it for other applications. In addition to the physical way, the behavior of the integrated circuit can be analyzed by creating a mathematical model of the basic and logical blocks (Martínez-Nieto et al., 2018). Over the last decade, several software products (such as: B2.Spice A/D Circuit, Every Circuit, Circuit Logix and others) have been created specifically for this purpose. Advanced in the present paper is a mathematical model of a driver circuit for a half-bridge DC-DC converter. The model gives us an information of the relationship between the logic blocks and the elements, which are forming the operating frequency - $f_{s w}$ during normal operation of the converter and protection modes at $\frac{1}{3} V_{c c}, \frac{2}{3} V_{c c}, \frac{1}{6} V_{c c}$.

\section{Mathematical model of half bridge DC - DC converter}

The study into the integrated circuit parameters is a set of measures used to obtain information about the change of physical parameters of the semiconductor element during the various modes of operation. The emitted temperature, the dissipated power, the internal potentials and currents, the resistance to external factors are the main parameters that must be monitored and, if possible, the information about them should be stored for a certain period of time. These parameters provide a basis for analyzing the behavior of the integrated circuit, under certain operating modes and electrical circuits affecting the structural integrity of the integrated circuit. There are dozens of methods and techniques for compiling algorithms for semiconductor element analysis. Integrated circuits with higher integration of electronic components are significantly more difficult to analyze. In order to make a simplified representation of an integrated circuit, it is necessary to study its structure in detail.The programming environment - MATLAB in electronics is used to simulate linearly with non-linear electronic circuits, filters, amplifiers, oscillators and others. Developed in the add-on product Simulink was a mathematical model of the half bridge self-oscillating driver - IRS2153. 


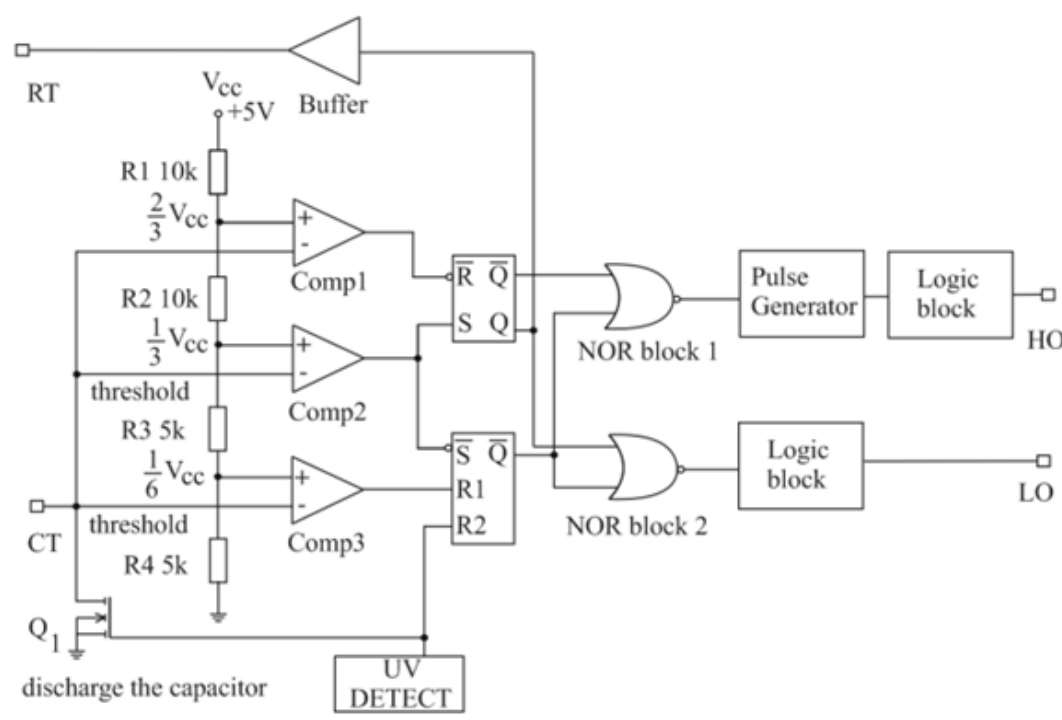

Fig.1 Block diagram of the main blocks of self-oscillating IRS2153 forming the switching frequency $f_{S w}$ and the logic blocks transmitting the signal to the output pins of the driver to High and Low side transistors.

The model was created to simulate the behavior of the integral circuit:1) nodal voltages 2)output waveforms of the pin LO,HO during $\left.\frac{1}{3} V_{c c}, \frac{2}{3} V_{c c}, \frac{1}{6} V_{c c}, 3\right)$ performance of the integrated components in the IC: triggers, comparators, voltage dividers and others. The mathematical model is composed of three level voltage divider, flip-flop trigger, two RS triggers, four level comparator, amplifier and BJT transistor. Most of the basic components in integral execution were developed by datasheet documents. The triggers were modeled with basic logic components - AND, OR, OFF-AND / OR and buffer circuits. The buffers between the triggers were modelled by the equation:

$$
i(t)=w_{1}(t) i_{1}(v(t))+w_{2}(t) i_{2}(v(t))
$$

where $w, i, v$ are buffer variables. Reproduce through a DC voltage block, DC motor, MOSFET transistors, diode and transformer blocks from the library Simscape, was the working principle of DC-DC converter. The model of IRS2153 generates control pulses to the low- and high-level transistors. The pulses applied to the control transistors are the same as if it were done by Pulse generator blocks. The implementation and simulation of an integrated circuit (in particular - a driver circuit) is significantly more time consuming and the probability of incorrect or inaccurate modeling considerably increases. In order to implement such a scheme, it is necessary to make a thorough analysis of its structure in order to clarify the relationship between their blocks (Karris, 2012). 


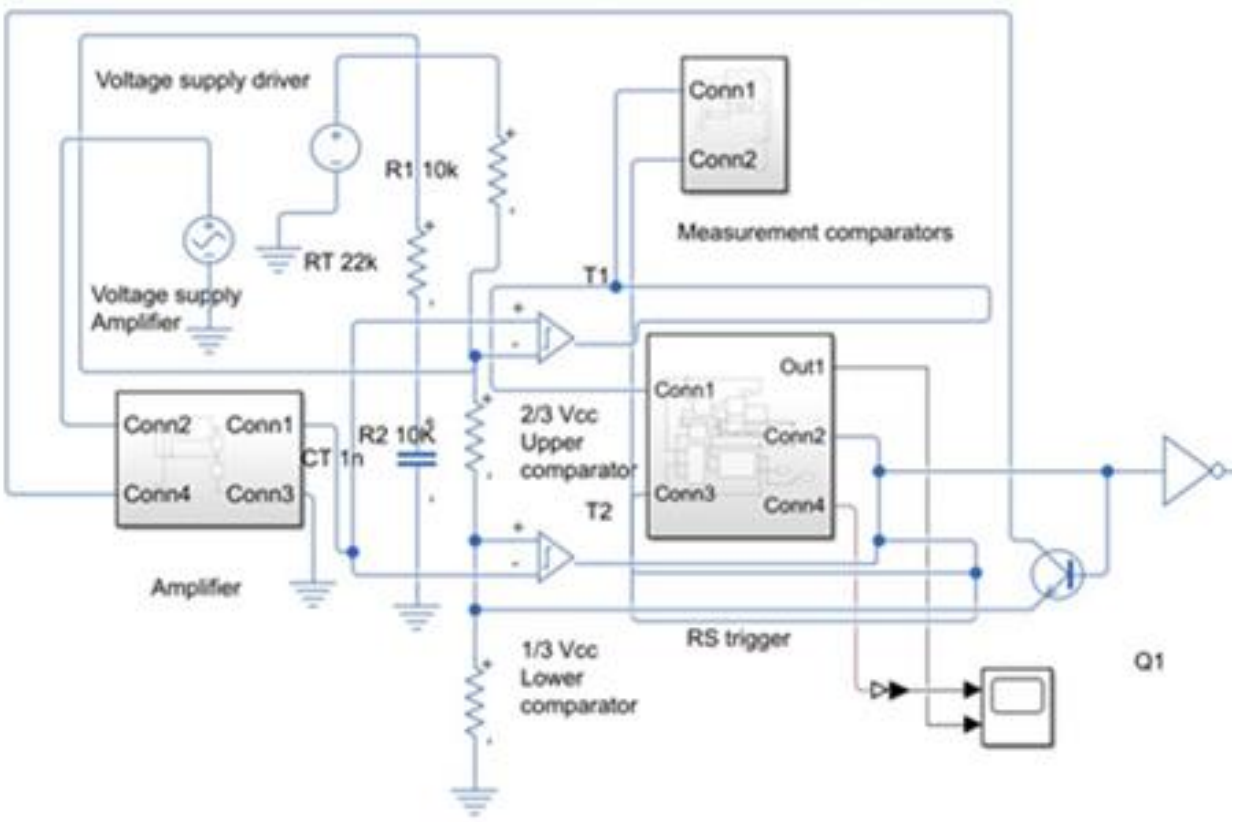

Fig.2 Block scheme of the mathematical model of IRS2153 in Simulink

The mathematical model allows us to trace the potentials of the main components of the driver circuit that directly affect the pins RT, CT during $\frac{1}{3} V_{c c}, \frac{2}{3} V_{c c}, \frac{1}{6} V_{c c}$, depending on the motor voltage received from the DC-DC converter`s feedback. The model produced conveys information about the currents and voltages flowing between the voltage divider, the comparators relative to the capacitor responsible for forming the operating frequency. This frequency, in turn, directly affects the generated pulses to the converter`s switches. For more in-depth analysis, a more complex equivalent circuit of the driver can be created. The components of the equivalent circuit can be modeled more easily in keeping with the data obtained from the current mathematical model (Enocksson, 2011). This model needs to be designed over a much longer time period to effectively simulate all the blocks of the integrated circuit.

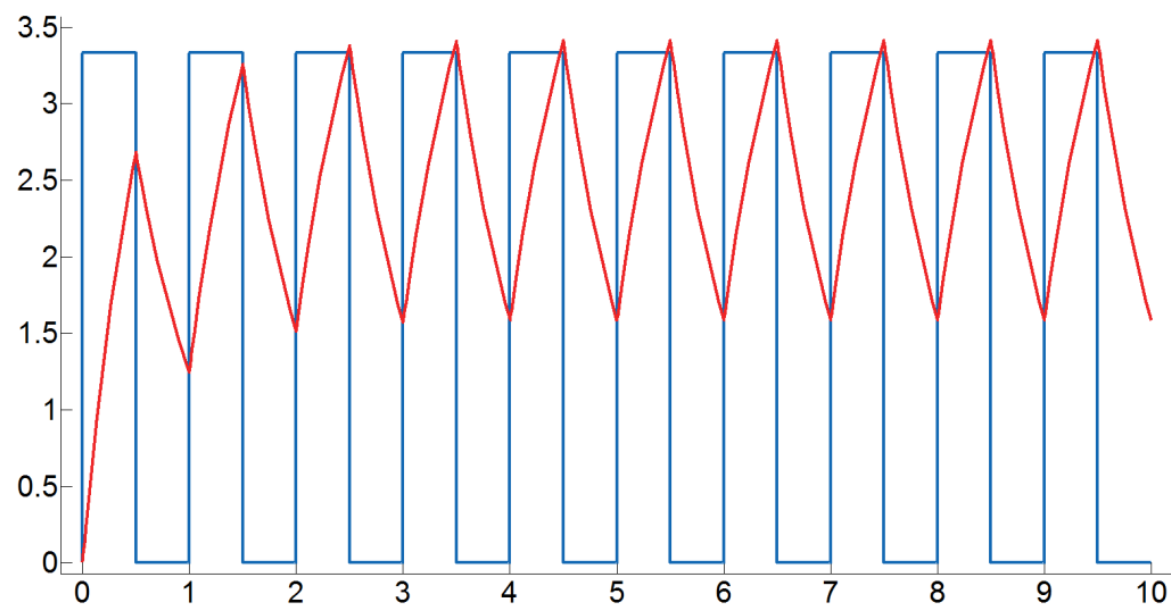

Fig.3 Time diagrams of the frequency capacitor $-\mathrm{C} 1$ and low side transistor 


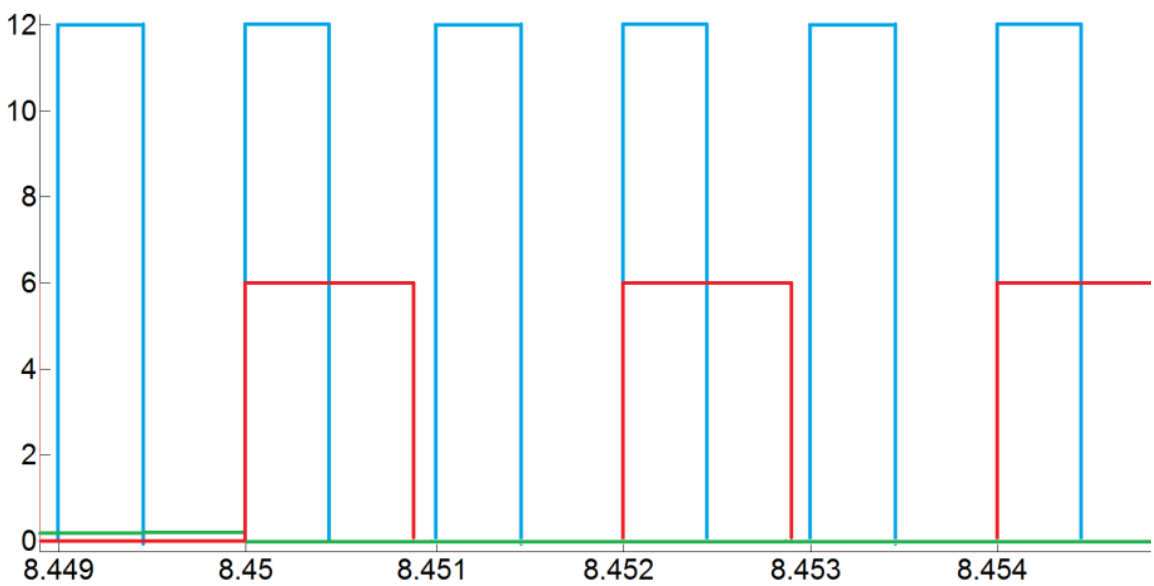

Fig.4 Time diagrams of the switch Q1 - during normal operation of the switch (blue), pre-emergency operation of the switch $-\frac{2}{3} V_{c c}$ (red) and operation in emergency mode $-\frac{1}{6} V_{c c}$ (green), when the operating voltage forming the pulse is reduced to 0 .

The mathematical model successfully represents the self-oscillating process associated with the driver circuit and the capacitor that forms the frequency - C1 (Santillan, 2018). Graphically, this is shown in Figure 2. In the following figure, we see a simulation of the integrated protection of the integrated circuit. At reference voltages equal to $\frac{2}{3} V_{c c}, \frac{1}{6} V_{c c}$, accordingly, the output pulses $\left(\frac{2}{3} V_{c c}-\right.$ high transistor, $\frac{1}{6} V_{c c}$ - both transistors) are reduced to zero. From the creation of this mathematical model of the driver circuit, we can conclude that MATLAB is suitable for simulating the nodal voltages of an equivalent driver circuit, build from digital components (Data sheet No.PD60062).

\section{Simulation of driver circuit in Infineon designer}

Infineon designer - this is the first software for online simulation of the behavior of integrated circuits. The product makes allowances for a thorough analysis of analog and digital integrated circuits. The simulation results include - Fourier analysis - used to identify the sources of parasitic vibrations in each part of the integrated circuit, Method with nodal potentials, Thermo-mechanical stress analysis (Niehoff et al., 2009), DC transient analysis, temperature analysis of the circuit at high frequencies and the occurrence of saturation in the inductive components and transient analysis (Chin et al., 1989). Set forth are the results of simulation of the IR2301 driver circuit, which in terms of construction is closest to the integrated circuits of the IR21XX series (Data sheet No.PD60201).
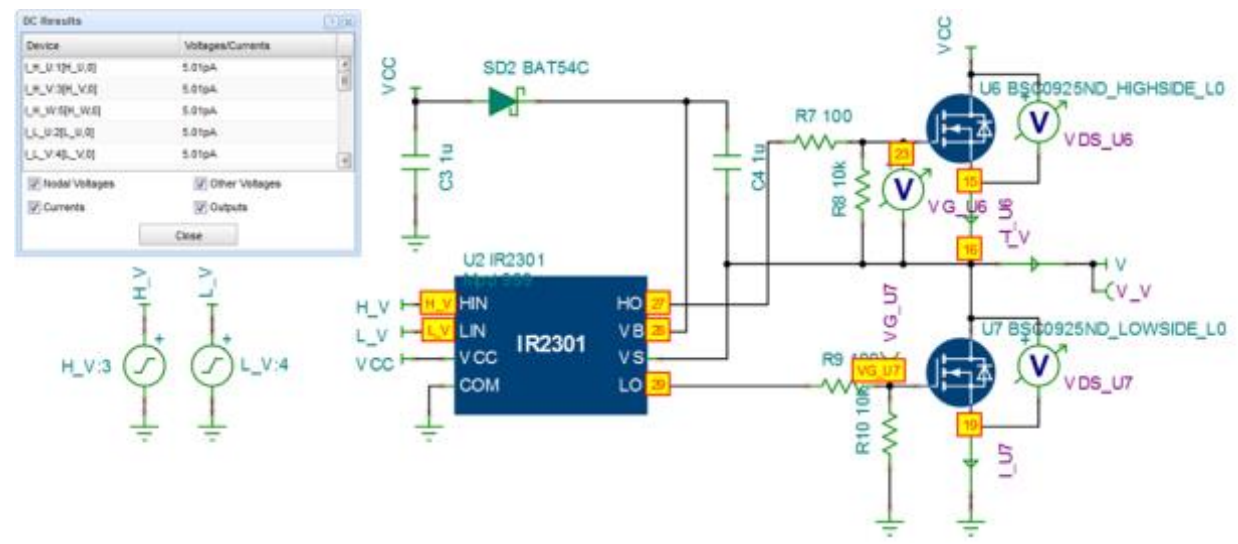

Fig.5 Simulation circuit of a driver circuit IR2301 in Infineon designer 
From the obtained results, we see that this simulation product provides for simulation of physical processes developed in the integrated circuit. Information on power dissipation, stress analysis and transient analysis will be useful in designing an effective DC-DC power converter. In addition, such data is useful when selecting a driver`s package, designing heatsink system and placing it on the PCB. Figure 6 depicts the main voltage and currents generated from the Infineon driver circuit. Disclosed in the next pictures is stress analysis giving information about power dissipation (Fig.7) alongside a Fast Fourier analysis (Fig.8).

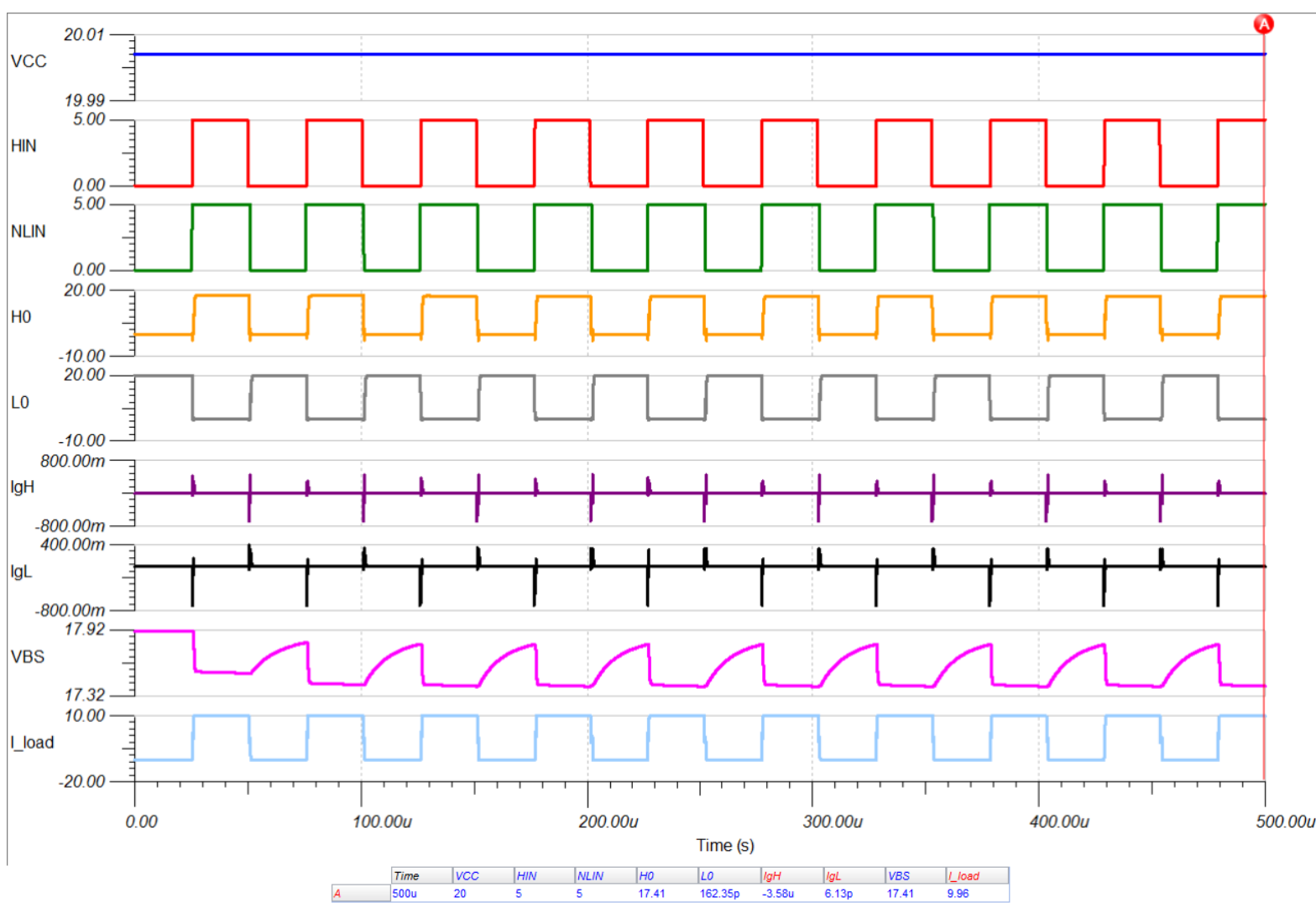

Fig.6 Waveforms of the main parameters of the driver Supply voltage of the driver (blue), output logic block High side Switch (red), output logic block - Low side Switch (green), Drive impulse High side Switch (yellow),

Drive impulse low side Switch (grey), Current during switching High side Switch (purple), Current during switching Low side Switch (black), Set frequency capacitor (pink), Load current (light blue) 


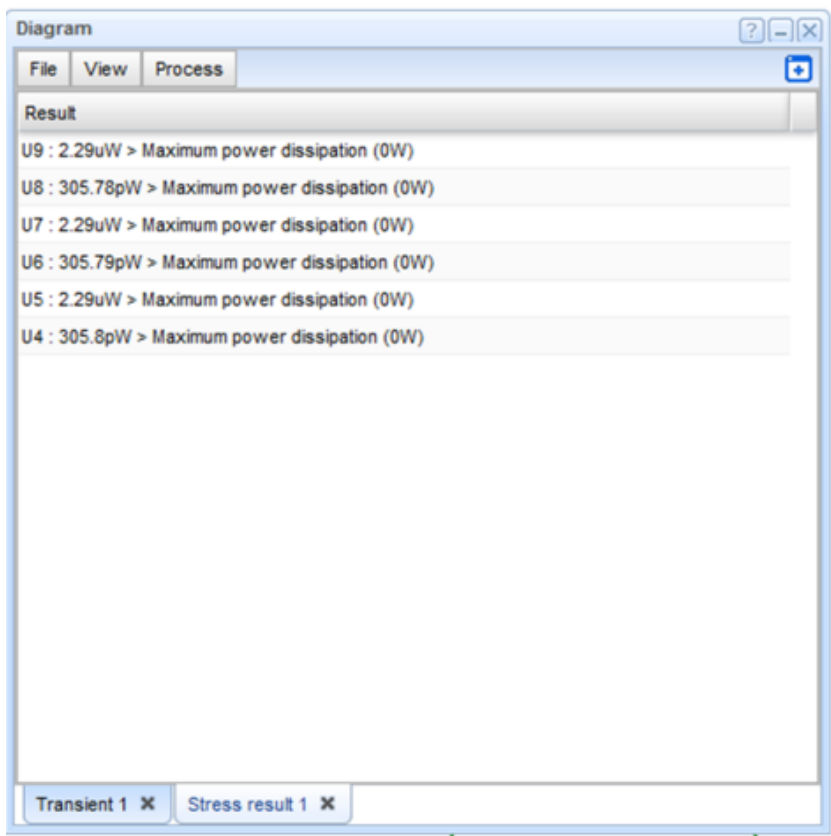

Fig.7 Termo-mechanical stress analysis results of the driver circuit

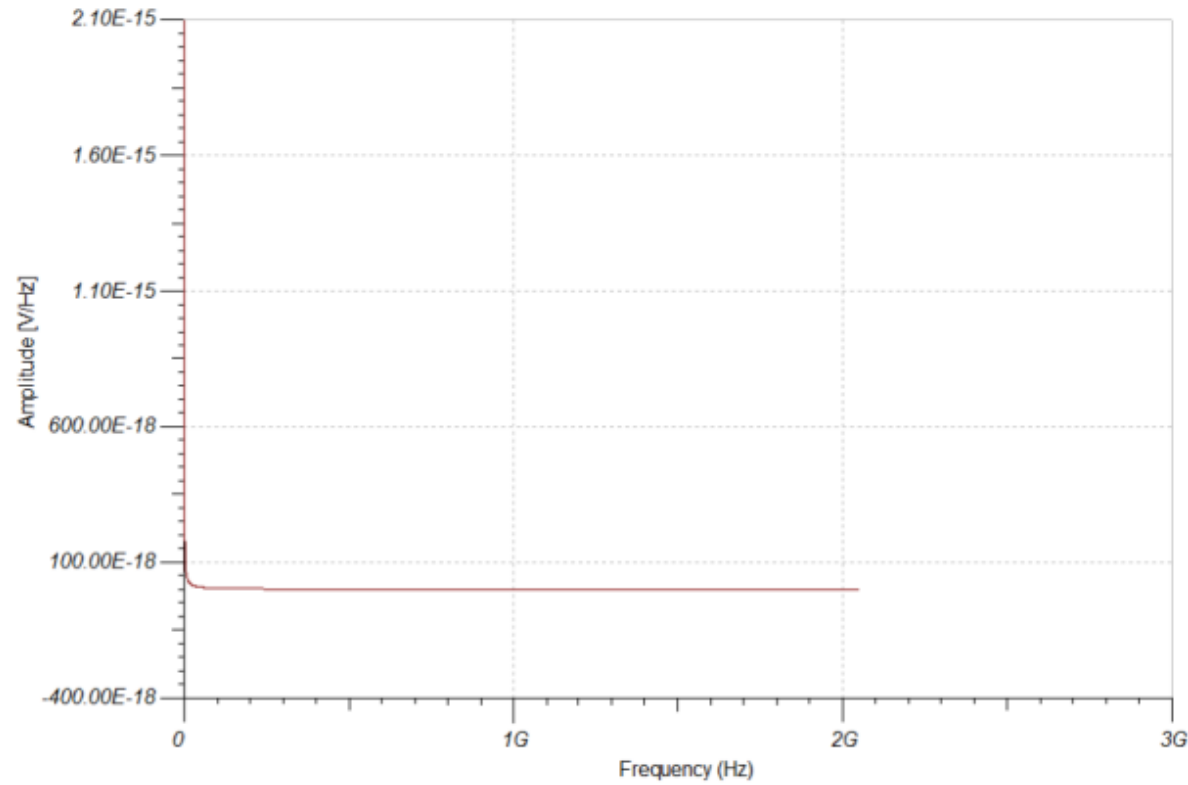

Fig.8 Fourier spectrum analysis of IR2301

The Fourier analysis is included for a more accurate perception and understanding of the maximum frequency resolution. The exponent of this parameter can be calculated by the formula (Wagstaff 2003):

$$
E X P=\frac{\log \left(\frac{f_{S}}{\operatorname{maxf} f_{\text {res }}}\right)}{\log (2.0)}
$$

where $f_{S}$ is sample frequency and $\max f_{\text {res }}$ is the maximum frequency resolution. 
The study into the output voltages and currents from the integrated circuit was accomplished on the basis of the transient analysis. The differential equation of a non-linear circuits (Yuan \& Opal ,2002) is given by the formula (Volterra functional series):

$$
\begin{gathered}
y(t)=\sum_{m=1}^{\infty} y_{m}(t) \\
y_{m}(t)=\int_{-\infty}^{\infty} \ldots \int_{-\infty}^{\infty} h_{m}\left(t, \tau_{1}, \ldots, \tau_{m}\right) x\left(\tau_{1}\right) \ldots x\left(\tau_{m}\right) d \tau_{1} \ldots d \tau_{m}
\end{gathered}
$$

, where $y_{m}$ is the $\mathrm{m}^{\text {th }}$ - order term of the Volterra series expansion of $y(t)$ and $h_{m}\left(t, \tau_{1}, \ldots, \tau_{m}\right)$ is the $\mathrm{m}^{\text {th }}$-order Volterra kernel.

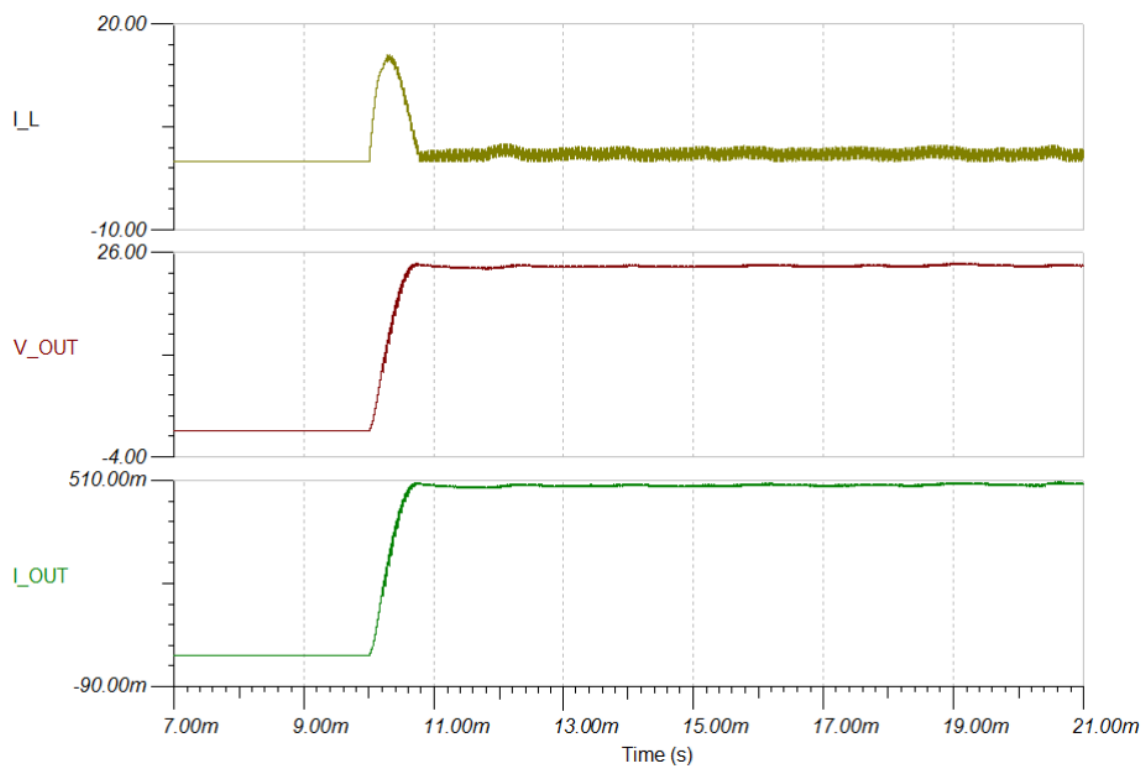

Fig.9 Transient analysis of IR2301 with RL-load equivalent to a DC motor

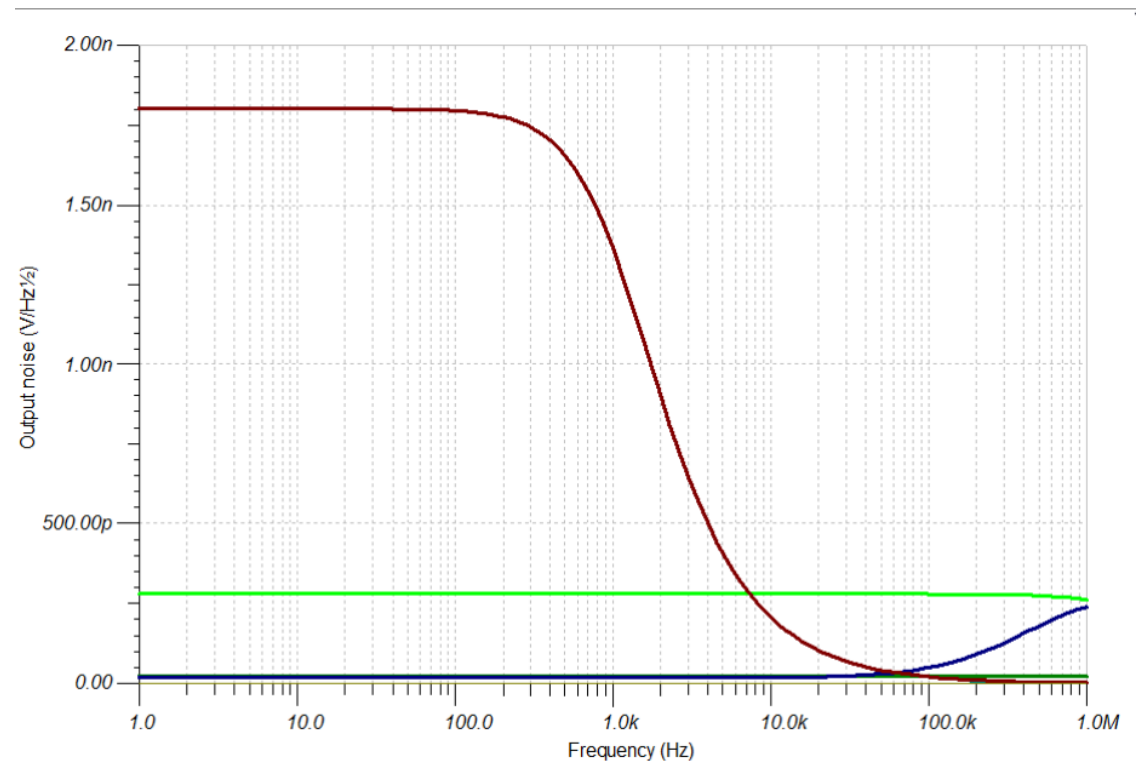

Fig.10 Noise analysis (Voltage per Herz) of IR2301 Voltage of frequency capacitor (dark red), output logic block - High side Switch (light green), output logic block - Low side Switch (green), Drive impulse High side Switch (blue)

There are also other software products that can help for more in-depth analysis and modeling of the driver circuits ( Analog devices www.analog.com/). 


\section{Simulation software for integrated circuits}

ADIsimRF - software developed by the American multinational semiconductor company - Analog devices. In general, this product represents easy-to-use RF signal chain calculator. The software contains libraries with components that include basic Boolean blocks, basic triggers circuits, basic amplifier circuits. All this circuits mathematically, are modeled by physical components produced from the company. ADIsimRF analyzes and provides information on the following parameters of the integrated circuit: input and output impedances, power and voltage gain, Noise waveforms, Output Power Backoff and others. Such a block is extremely suitable for analyzing and compiling mathematical models of blocks building integrated circuits (Caspers \& Kowina, 2013).

Software platforms based on finite element analysis such as Comsol and Ansys can make simulation on many physic processes, which develop into a semiconductor integrated circuit (Shanmugavel,2013). Modeling such a product is significantly more difficult and requires more in-depth knowledge related to the construction of integrated circuits. It is also necessary to spend more time modeling the materials from which objects are built and the physical processes that develop in them. Such software allows analysis of thermal behavior of the IC, the noises and the voltage stress between the different layers of the IC. Such software has capabilities for in-depth analyses of the thermal behavior of the integrated circuits.

Heat transfer equation of a semiconductor integrated circuit:

$$
k_{x}^{(i)} \frac{\partial^{2} \psi^{(i)}}{\partial x^{2}}+k_{y}^{(i)} \frac{\partial^{2} \psi^{(i)}}{\partial y^{2}}+k_{z}^{(i)} \frac{\partial^{2} \psi^{(i)}}{\partial z^{2}}=0
$$

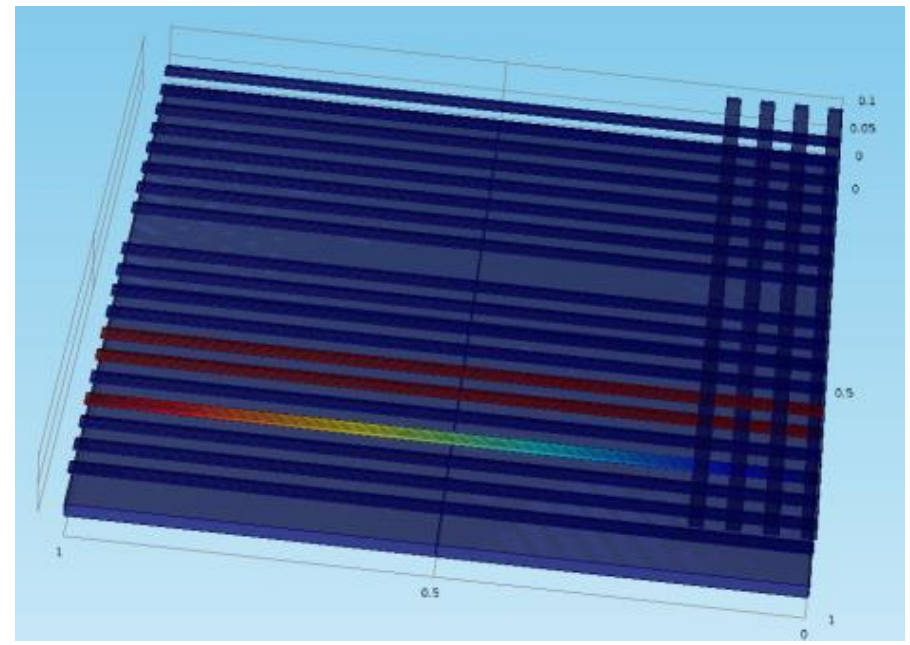

Fig.11 Model of integrated circuit in Comsol.

\section{5 . Conclusion}

Advanced in the present paper is a mathematical model of an integrated circuit - a driver for a halfbridge DC-DC converter. The model allows us to analyze the operation of the main blocks of the integrated circuit. Obtained, accordingly, is useful information about the interaction of IC's comparators with the elements forming the frequency of the converter. Released, additionally, via the mathematical model in the Infineon designer`s software, is information about the physical parameters to help determine the maximum frequency resolution, stress analysis results (power dissipation in the integrated circuit), peak and switching currents.

\section{Gratitude}

I would like to express my gratitude for the realization of my practical training under the Erasmus+ program at the University of Ghent. I want to express my deepest gratitude to Prof. Alex Van den Bosque for the many practical tips and advice in the field of science. 


\section{References}

Stievano I. S., Canavero F. G. ,Maio I. A.(2003). On the behavioral modeling of integrated circuit output buffers, Electrical Performance of Electrical Packaging (IEEE Cat. No. 03TH8710), Princeton, NJ, USA. pp. 281-284. https://doi.org/10.1109/EPEP.2003.1250050

Lee, H. H. S., \& Chakrabarty, K. (2009). Test challenges for 3D integrated circuits. IEEE Design \& Test of Computers, 26(5), 26-35. https://doi.org/10.1109/MDT.2009.125

Martínez-Nieto A., Medrano N., Sanz-Pascual M. T., \& Calvo B.(2018). An Accurate Analysis Method for Complex IC Analog Neural Network-Based Systems Using High-Level Software Tools. IEEE 9th Latin American Symposium on Circuits \& Systems (LASCAS). https://doi.org/10.1109/LASCAS.2018.8399902

Yuan, F., \& Opal, A. (2002). An efficient transient analysis algorithm for mildly nonlinear circuits. IEEE Transactions on Computer-Aided Design of Integrated Circuits and Systems, 21(6), 662-673. https://doi.org/10.1109/TCAD.2002.1004310

Lee, C. C., Palisoc, A. L., \& Min, Y. J. (1989). Thermal analysis of integrated circuit devices and packages. IEEE transactions on components, hybrids, and manufacturing technology, 12(4), 701-709. https://doi.org/10.1109/33.49036

IR2301 High and low side driver Data sheet No.PD60201. Infineon technologies AG. Retrieved May 10, 2021 from https://www.infineon.com

IR2153 Self-oscillating half-bridge driver Data sheet No.PD60062. Infineon technologies AG. Retrieved May 10, 2021 from https://www.infineon.com

Shanmugavel, A. (2013). Robust Design for Integrated Circuits.

ADIsimRF Signal Chain Calculator. Analog devices. Retrieved May 10, 2021 from https://www.analog.com/en/design-center/adisimrf.html ADIsimRF Signal Chain Calculator

Wagstaff, R. D. (2003). U.S. Patent No. 6,545,454. Washington, DC: U.S. Patent and Trademark Office.

Niehoff, K., Schreier-Alt, T., Schindler-Saefkow, F., Ansorge, F., \& Kittel, H. (2009). Thermomechanical stress analysis. In 2009 European Microelectronics and Packaging Conference (pp. 1-5). IEEE.

Enocksson S. (2011) Modeling in MathWorks Simscape by building a model of an automatic gearbox UPTEC STS11 017

Karris, S. T. (2012). Electronic Devices and Amplifier Circuits: With MATLAB. Orchard Publications.

Caspers F., \&Kowina P. (2013). RF Measurement Concepts Advanced Accelerator Physics, Trondheim, Norway, 19-29 August 2013, edited by W. Herr, CERN-2014-009 (CERN, Geneva, 2014) pp.105-110 